\title{
Cooperative pursuit with sensing limitations
}

\author{
Shaunak D. Bopardikar
}

\author{
Francesco Bullo
}

\author{
João P. Hespanha
}

\begin{abstract}
We address a discrete-time pursuit-evasion problem involving multiple pursuers and a single evader in an unbounded, planar environment in which each player has limited-range sensing. The evader appears at a random location in a bounded region and moves only when sensed. We propose a sweep-pursuit-capture strategy for a group of at least three pursuers and determine a lower bound on the probability of capture for the evader. This bound is a function of the pursuer formation and independent of the initial evader location and the evader strategy. We then propose a novel cooperative pursuit algorithm and show that the problem is reduced to one with unlimited sensing. We provide an upper bound on the time for our pursuit strategy to succeed. The final capture is achieved by using the established algorithm SPHERES. Our results show that on the basis of maximizing the probability of evader capture per pursuer, the pursuers should search the bounded region as a single group (conjoin) rather than to divide the region into smaller parts and search simultaneously in smaller groups (allocate).
\end{abstract}

\section{INTRODUCTION}

The game of pursuit can be posed as to determine a strategy for a team of pursuers to capture an evader in a given environment. By capture, we mean that the evader and some pursuer are at the same location after a finite time. The aim of the pursuers is to capture an evader for any evader trajectory. The evader wins the game if it can avoid capture indefinitely. All the players have identical motion capabilities.

\section{A. Related Work}

The continuous time version of this problem has been studied by Ho et al. [1], Lim et al. [2] and Pachter [3] to cite a few. Recently, significant attention has been received by the discrete-time version of the game. The paper by Sgall [4] gives sufficient conditions for a single pursuer to capture an evader in a semi-open environment. This strategy has been extended to multiple pursuers in an unbounded environment by Kopparty and Ravishankar [5]. The algorithm SPHERES enables the pursuers to capture an evader initially located inside their convex hull. Alonso et al. [6] and Alexander et al. [7] propose strategies so that the pursuer can reduce the distance between itself and the evader to a finite, nonzero amount after finite time steps. The game has also been studied in different types of bounded environments, e.g., circular environment by Alonso et al. [6], curved environments by LaValle et al. [8]. Visibility-based pursuit evasion has

\footnotetext{
Shaunak D. Bopardikar and Francesco Bullo are with the Department of Mechanical Engineering, University of California at Santa Barbara, Santa Barbara, CA 93106, USA, \{shaunak, bullo\}eengineering.ucsb.edu

João P. Hespanha is with the Department of Electrical and Computer Engineering, University of California at Santa Barbara, Santa Barbara, CA 93106, USA, hespanhadece.ucsb.edu
}

been studied by Guibas et al. [9], Sachs et al. [10] and in polygonal environments by Isler et al. [11].

Each of above mentioned works proposes strategies which require that the pursuers have unlimited sensing capacity. A more realistic assumption is to introduce sensing limitations for the pursuers and the evader. In this context, Gerkey et al. [12] have studied a version of visibility limited to an angle, instead of the entire region. Isler et al. [13] have considered the problem on a graph, with the visibility of the pursuer limited to nodes adjacent to the current node of a pursuer. A framework which uses probabilistic models for sensing devices for the agents can be found in the works of Hespanha et al. [14] and Vidal et al. [15].

\section{B. Contributions}

We consider the case of sensing capabilities restricted to a closed disc of a given sensing radius around the current positions of the players. The motion of each player is confined to a closed disc of a given stepping radius around its current position. The game is played in an unbounded, planar environment. The evader is assumed to be initially located in a bounded region and follows a reactive rabbit model [13], i.e., moves only when detected. We propose a strategy for the pursuers, comprising of three phases sweep, pursuit and capture. In the sweep phase, the pursuers search the bounded region in a proposed formation. They succeed when they detect the evader inside a special capture region, which we characterize for the pursuer formation. We show that using our sweep strategy, the probability of pursuer success is a function of the pursuer formation and independent of the initial evader location. Next, we propose a cooperative pursuit strategy for the pursuers to confine the appropriately-sensed evader within their sensing discs. We show that using this pursuit strategy, the problem is converted into a previously-studied problem of pursuit-evasion with unlimited sensing. We also give an upper bound on the time for our trapping strategy to converge. The final capture is achieved by using the established algorithm SPHERES [5].

Of all proposed pursuer formations, we define a limiting formation that gives maximum probability of evader capture for a given number of pursuers. For this limiting formation, we analyze the pursuers' decision to search the bounded region as a single group (conjoin) or to divide the region into smaller parts and search simultaneously in smaller groups (allocate).

\section{Biological Motivation}

The inspirations for the strategies proposed in this paper have been derived from aspects of animal behavior. It is 
well known that predators hunt as a conjoined group when it is less efficient to hunt alone. This behavior is also observed when the prey is large or can move as fast as the predators [16]. Further, predators show an inclination towards specialized behavior by maintaining a fixed formation during search and capture of preys [17]. Such specializations suggest that there may be configurations that are preferred during group hunting. Also, in presence of sensing limitations, groups tend to maintain spacing between each other that is regulated by their sensory capabilities [18]. These facts give us some additional hints towards selecting capture-conducive predator formations.

\section{Organization}

The problem assumptions and its mathematical model are presented in Section II. In Section III, we describe the pursuer formations for the search-pursuit-capture strategies and give the corresponding results. The respective proofs are given in Section IV. Section V deals with the analysis for determining whether the pursuers should allocate or conjoin.

\section{Problem Set-UP}

We assume that there exists a finite, region, $\mathcal{G} \subset \mathbb{R}^{2}$, where an evader appears at a random location. The motion and sensing abilities of the evader are restricted to closed discs of radii $r_{\text {step }}$ and $r_{\text {sens }}$ respectively, around the current evader position. We have a total of $n$ pursuers, who have the same motion and sensing capabilities as those for the evader. We assume a discrete-time model with alternate motion of the evader and the pursuers. The pursuers have complete communication between themselves, i.e., they can communicate the location of a sensed evader as well as their own position with respect to a fixed, global reference frame, among themselves. The evader wins if it can avoid being captured indefinitely. We seek pursuer strategies to capture the evader.

Define $\mathbb{R}^{2}{ }_{\mathrm{e}}=\mathbb{R}^{2} \cup \phi$, where $\mathbb{R}^{2}$ is the unbounded, planar environment and $\phi$ is the null element. Here, the null element refers to the fact that during sensing, the measurement of the position of an evader may not be available to all pursuers. Let $e[t]$ and $p_{k}[t]$ denote the absolute positions of the evader and the $k^{t h}$ pursuer respectively, at time $t$. Here, $k$ takes all values in $\{1, \ldots, n\}$. The equations of motion in discretetime can be written as,

$$
\begin{aligned}
e[t+1] & =e[t]+u^{e}\left(e[t],\left\{y^{p_{k}}[t]\right\}\right), \\
p_{k}[t+1] & =p_{k}[t]+u^{p_{k}}\left(e[t], y^{e}[t+1], p[t]\right),
\end{aligned}
$$

where $y^{p_{k}}[t] \in \mathbb{R}^{2}$ is the measurement of the $k^{t h}$ pursuer position taken by the evader at the $t^{t h}$ time instant and $y^{e}[t+$ $1] \in \mathbb{R}^{2}{ }_{\mathrm{e}}$ is the measurement of the evader position taken by some pursuer at the $(t+1)^{t h}$ time instant. These are given by

$$
y^{p_{k}}[t]= \begin{cases}p_{k}[t], & \text { if }\left\|p_{k}[t]-e[t]\right\| \leq r_{\text {sens }} \\ \phi, & \text { otherwise. }\end{cases}
$$

Similarly,

$$
y^{e}[t+1]= \begin{cases}e[t+1], & \text { if for some } k \in\{1, \ldots, n\} \\ & \left\|p_{k}[t]-e[t+1]\right\| \leq r_{\text {sens }} \\ \phi, & \text { otherwise }\end{cases}
$$

The functions $u^{e}: \mathbb{R}^{2}{ }_{\mathrm{e}} \times \mathbb{R}^{2} \rightarrow \mathbb{R}^{2}$ and $u^{p_{k}}: \mathbb{R}^{2}{ }_{\mathrm{e}} \times \mathbb{R}^{2}{ }_{\mathrm{e}} \times$ $\mathbb{R}^{2} \rightarrow \mathbb{R}^{2}$ are termed as strategies for the evader and pursuer respectively. Due to the reactive rabbit model for the evader, $u^{e}=0$ until the evader is sensed by the pursuers for the first time. The constraint on the maximum step size gives,

$$
\left\|u^{e}\right\|,\left\|u^{p_{k}}\right\| \leq r_{\text {step }} .
$$

Let $t_{0}$ denote the time at which the evader is detected by the pursuers. We seek sufficient conditions on $e\left[t_{0}\right]$ relative to positions of the pursuers $p_{k}\left[t_{0}\right]$ and a corresponding pursuers strategy $u^{p_{k}}$ so that the pursuers will capture the evader for any evader strategy $u^{e}$.

We now define the notion of capture as follows.

Definition II.1 (Capture notion) The evader is said to be captured by the pursuers if for any evader strategy $u^{e}$, some pursuer is at the same position as the evader after a finite time.

We also introduce the notion of trapping an evader as follows.

Definition II.2 (Trapping notion) The evader is said to be trapped within the sensing radii of the pursuers if for any evader strategy $u^{e}$, the motion disc of the evader is completely contained within the union of the sensing discs of the pursuers after a finite time.

\section{The Algorithms And MAIN Results}

We propose a pursuer strategy with three phases - sweep, pursuit and capture. In this section, we describe the algorithms in each phase and the corresponding results.

Let the sensing radius be $\kappa$ times the stepping radius. We assume throughout our problem that $\kappa$ is large enough: typically greater than $2 \sqrt{2}$. We define the following pursuer formation.

Definition III.1 (Trapping chain) $A$ group of $n \geq 3$ pursuers $\left\{p_{1}, \ldots, p_{n}\right\}$ are said to be in a trapping chain formation if

(i) $p_{1}, \ldots, p_{n}$ are at the vertices of a convex polygon such that,

$$
\left\|p_{1}-p_{n}\right\|=\max _{k \in\{2, \ldots, n\}}\left\|p_{1}-p_{k}\right\|, \text { and, }
$$

(ii) for all $k \in\{1, \ldots, n-1\}$,

$$
\left\|p_{k}-p_{k+1}\right\| \leq 2 r_{\text {step }} \sqrt{\kappa^{2}-4}
$$

An example of a trapping chain is shown in Figure 1. We define the capture region for a trapping chain as under. 


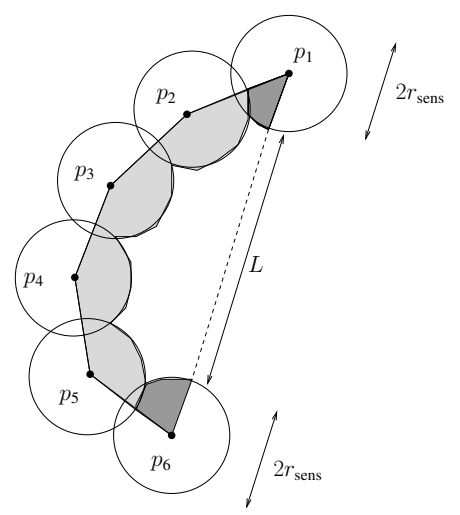

Fig. 1. A trapping chain. The circles around the pursuers represent their sensing discs.

$$
\mathcal{S}[t]=\bigcup_{k \in\{2, \ldots, n-1\}} \mathcal{B}_{p_{k}}\left(r_{\text {sens }}\right) \cap \mathrm{Co}\left\{p_{1}, \ldots, p_{n}\right\}[t] .
$$

The lightly shaded region in Figure 1 is the proposed capture region $\mathcal{S}$ for the trapping chain.

There is a chance that the evader can step into a region such that the pursuers trap it within their sensing radii. So we define an extended capture region for the trapping chain as under,

$$
\mathcal{S}^{e}[t]=\bigcup_{k \in\{1, \ldots, n\}} \mathcal{B}_{p_{k}}\left(r_{\text {sens }}\right) \cap \operatorname{Co}\left\{p_{1}, \ldots, p_{n}\right\}[t] .
$$

The darkly shaded region along with the lightly shaded region in Figure 1 is the extended capture region $\mathcal{S}^{e}$ for the trapping chain.

The following are the strategies in each phase.

\section{A. Sweep Phase}

The pursuers begin by placing themselves in a trapping chain formation. In the sweep phase, their aim is to sense an evader within the capture region of the trapping chain. For this purpose, we propose that the pursuers sweep $\mathcal{G}$ in the direction of the outward normal to $p_{1} p_{n}$, with respect to the convex hull of the pursuers. We demonstrate our result for a square region $\mathcal{G}$ of length $b$. But it would be clear from our approach that the result is valid for any bounded environment. For a trapping chain shown in Figure 1, we define,

$$
L=\left\|p_{1}-p_{n}\right\|-2 r_{\text {sens }} .
$$

As the pursuers move in the direction described earlier, they clear a rectangular strip of length $b$ and width of at most $L+4 r_{\text {sens }}$, of which the favorable length is $L$. The sweeping policy for the pursuers is as follows.

(i) Choose the first rectangular strip at a random distance $l_{0}$ from one edge of $\mathcal{G}$ and sweep it length-wise. The distance $l_{0}$ is a uniform random variable taking values in the interval $\left[-2 r_{\text {sens }}, L+2 r_{\text {sens }}\right]$. Here, negative $l_{0}$ implies that some of the pursuers may begin sweeping from outside the region $\mathcal{G}$. (ii) Form a sweeping path for $\mathcal{G}$ and sweep along adjacent strips as shown in Figure 2.

The shaded region in Figure 2 refers to the area that would fall in the proposed capture region $\mathcal{S}$. Now we are interested in determining the probability that an evader falls in the shaded region in Figure 2. That is given by the following result.

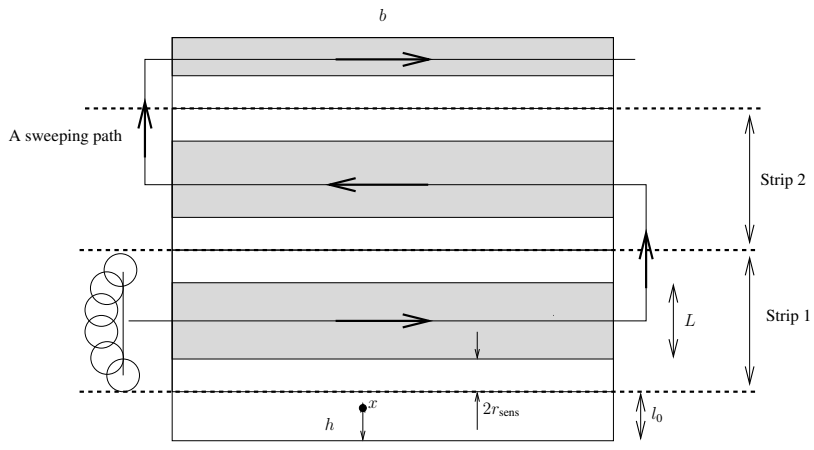

Fig. 2. A sweeping path

Theorem III.2 (Sweep property) For an evader located anywhere in $\mathcal{G}$, the probability $P$ of detecting it inside $\mathcal{S}$ for a group of pursuers in a trapping chain, using the sweeping policy, is given by

$$
P=\frac{L}{L+4 r_{\mathrm{sens}}},
$$

where $L$ is defined in equation (2).

Notice that the probability of pursuer success $P$ depends only on the number of pursuers $n$ and the relative pursuer locations. We shall refer to it as $P(n)$ from now on. We will use the result in Theorem III.2 in Section V. The pursuers win when the evader is detected in $\mathcal{S}$ by the pursuers. Otherwise, the evader is scared away and lost forever.

\section{B. Pursuit phase: algorithm TRAP}

Once an evader has been detected within the proposed capture region at time $t_{0}$, the pursuers need to ensure that the evader is trapped within their sensing ranges. For this purpose, we propose the following algorithm. At each time step $t \geq t_{0}$,

(i) While $e[t+1] \notin \mathcal{S}^{e}[t]$, the pursuers move towards the circumcenter $O$ of $\triangle p_{1}\left[t_{0}\right] e\left[t_{0}\right] p_{n}\left[t_{0}\right]$ with maximum step size.

(ii) Otherwise, one of the pursuers which senses the evader directly, moves with maximum step towards the evader and the others move parallel to that pursuer with the maximum step size.

The notation $\triangle X Y Z$ denotes the triangle formed by points $X, Y$ and $Z$. One such move is shown in Figure 3. In case (i) of the algorithm, note that the pursuers may not sense the evader in all the subsequent moves. But the intuition is that the pursuers will encircle the evader by completing the chain around it and trap it within the overlapping chain. Then the 
pursuers can shrink the chain around the evader. We define trapping time $t^{*}$ as the time taken by the pursuers to trap the evader within their sensing radii. We have the following result.

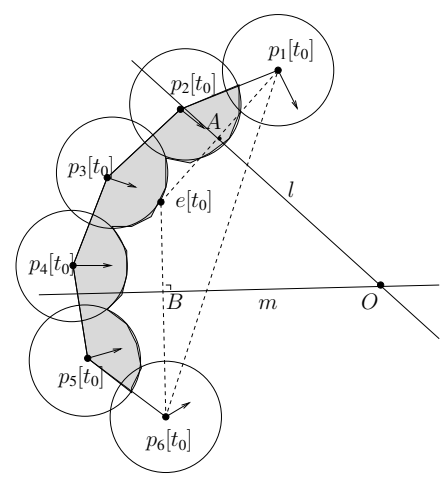

Fig. 3. Algorithm TRAP. The circles around the pursuers represent their sensing discs.

Theorem III.3 (TRAP) Starting from a trapping chain formation, if $e\left[t_{0}\right] \in \mathcal{S}\left[t_{0}\right]$, then

(i) the pursuers trap the evader within their sensing radii and their convex hull using algorithm TRAP.

(ii) The trapping time $t^{*}$ satisfies,

$$
t^{*} \leq \max _{k \in\{1, \ldots, n\}}\left\lceil\frac{\left\|p_{k}[0]-O\right\|}{r_{\text {step }}}\right\rceil,
$$

where $O$ is the circumcenter of $\triangle p_{1}\left[t_{0}\right] e\left[t_{0}\right] p_{n}\left[t_{0}\right]$.

\section{The Capture phase}

Once the evader is captured within the sensing ranges of the pursuers, the pursuers now have access to the next position of the evader at the present time instant. So the problem reduces to one having unlimited sensing capabilities for the pursuers. A capture strategy for this phase is algorithm SPHERES proposed by Kopparty and Ravishankar [5], which is being reproduced here for clarity and completeness. Let the time at the end of the pursuit phase be $\left(t_{0}+t^{*}\right)$.

- Each pursuer $p_{k}$ initially selects (by communication) a point $C_{k}$ such that,

- $p_{k}\left[t_{0}+t^{*}\right]$ lies on the line segment $C_{k} e\left[t_{0}+t^{*}\right]$ and

- The connected component of

$$
\mathbb{R}^{2} \backslash \cup_{k=1}^{n} \mathcal{B}_{C_{k}}\left(\left\|C_{k}-p_{k}\left[t_{0}+t^{*}\right]\right\|\right)
$$

that contains $e\left[t_{0}+t^{*}\right]$ is bounded. Here $\mathbb{R}^{2}$ refers to the entire unbounded environment. This is illustrated in Figure 4(a).

- For every pursuer, choose $p_{k}[t+1]$ on line joining $e[t+$ 1] and $C_{k}$ such that $\left\|p_{k}[t+1]-e[t+1]\right\|$ is minimized, subject to $\left\|p_{k}[t+1]-p_{k}[t]\right\| \leq r_{\text {step }}$. This move is shown in Figure 4(b).

Theorem III.3 shows that use of algorithm TRAP in the pursuit phase leads to the evader being trapped and inside the convex hull of the pursuers. Thus, final capture follows
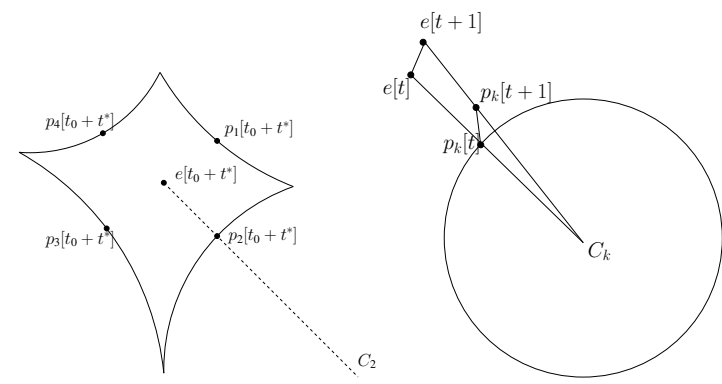

(a) Illustrating selection of (b) Illustrating a pursuer move points $C_{j}$

Fig. 4. Algorithm SPHERES

from the following theorem, the proof of which can be found in [5].

Theorem III.4 (SPHERES [5]) Assume the evader lies within the convex hull of the pursuers. If every pursuer follows the algorithm SPHERES, then

(i) the distance between every pursuer and the evader never increases at the end of every pursuer move.

(ii) The evader will be captured in finite number of steps.

Thus, once the evader is trapped within the sensing ranges of the pursuers, it would remain trapped within their sensing ranges at the end of every move using algorithm SPHERES. The capture phase is now complete.

\section{Proofs of the Main Results}

The main results in Section III are proved in this section. Proof of Theorem III.2: Let the evader be located at any point $x \in \mathcal{G}$ as shown in Figure 2. Let its distance from the lower edge be $h$. The event that $x$ would lie in the shaded region is given by $l_{0}+2 r_{\text {sens }}<h$ or $l_{0}-2 r_{\text {sens }}>h$. Thus, if we consider the interval $\left[-2 r_{\text {sens }}, L+2 r_{\text {sens }}\right]$, where $l_{0}$ takes values, the favorable interval is of length $L$. Thus, the probability of success for the pursuers is equivalent to determining the ratio of the lengths of the favorable interval, i.e., $L$ to the total interval, i.e., $L+4 r_{\text {sens. Hence, the result }}$ follows.

Remark IV.1 It is worthwhile to mention here that the probability of success for the proposed sweeping policy for the pursuers is independent of the evader's location $x$. Thus, the optimal policy for the evader in the present framework is to locate itself initially with a uniform probability density in $\mathcal{G}$.

To prove Theorem III.3, we first state the following properties of a trapping chain which follow from the definitions of trapping within sensing radii and of the trapping chain.

Lemma IV.2 (Trapping chain properties) If $e[t] \in \mathcal{S}[t]$, then the following statements hold.

(i) If $\operatorname{dist}\left(e[t], \overline{p_{k}[t] p_{k+1}[t]}\right)>r_{\text {step }}$, for all $k \in$ $\{1, \ldots, n-1\}$, then the evader cannot step outside $\mathrm{Co}\left\{p_{1}[t], \ldots, p_{n}[t]\right\}$ by crossing $\overline{p_{k}[t] p_{k+1}[t]}$. 
(ii) If $\operatorname{dist}\left(e[t], \overline{p_{k}[t] p_{k+1}[t]}\right) \leq r_{\text {step }}$ for some $k \in$ $\{1, \ldots, n-1\}$, then the evader is trapped within the sensing radii of pursuers $p_{k}$ and $p_{k+1}$.

Proof of Theorem III.3: If $\operatorname{dist}\left(e[0], \overline{p_{k}\left[t_{0}\right] p_{k+1}\left[t_{0}\right]}\right) \leq r_{\text {step }}$ for some $k \in\{1, \ldots, n-1\}$, then the evader is already trapped within the sensing ranges of the pursuers, from part (ii) of Lemma IV.2. So let $\operatorname{dist}\left(e\left[t_{0}\right], \overline{p_{k}\left[t_{0}\right] p_{k+1}\left[t_{0}\right]}\right)>r_{\text {step }}$, for every $k \in\{1, \ldots, n-1\}$. If $e[t+1] \in \mathcal{S}^{e}[t]$, for any $t \geq t_{0}$, then the pursuers would use part (i) of the algorithm TRAP and the result follows.

Finally, if $e\left[t_{0}+1\right] \notin \mathcal{S}\left[t_{0}\right]$, then the pursuers compute

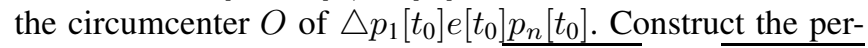
pendicular bisectors, $l$ and $m$ of $\overline{e\left[t_{0}\right] p_{1}\left[t_{0}\right]}$ and $\overline{e\left[t_{0}\right] p_{n}\left[t_{0}\right]}$ respectively, as shown in Figure 3. Any point on the side opposite to $e\left[t_{0}\right]$ of the lines $l$ and $m$ can be reached faster by $p_{1}$ and $p_{n}$ respectively. Since all the other pursuers are moving towards $O$, the overlap between their sensing discs increases at each step. Thus, the motion of the evader is confined to the quadrilateral $e\left[t_{0}\right] A O B$, which is cleared by the pursuers in finite time. The best path for the evader to get out of the convex hull is to move along $e\left[t_{0}\right]-O$ with maximum step size. Since $r_{\text {sens }}>r_{\text {step }}$, the sensing discs of pursuers $p_{1}$ and $p_{n}$ overlap before the evader can reach $O$, thus closing the trapping chain around the evader. Note that the evader is within the convex hull of the pursuers at the end of every pursuer move.

The bound in equation (2) is the time taken by the furthest pursuer (and hence all the pursuers) to reach point $O$. Thus, clearly at the end of this time, the sensing discs of the pursuers would have covered the interior of their convex hull. Thus, after at $\operatorname{most} \max _{k \in\{1, \ldots, n\}}\left\lceil\frac{\left\|p_{k}\left[t_{0}\right]-O\right\|}{r_{\text {step }}}\right]$ steps, the evader's motion circle would be contained within the sensing radii of the pursuers.

For the capture phase, we refer the reader to [5].

\section{Allocate or Conjoin?}

Our analysis in the previous sections sheds some light onto the trade-offs that agents evaluate when deciding between allocating a task by dividing into smaller groups and performing the task as a conjoined group. We explore some of these trade-offs in what follows.

Given a total of $k n$ pursuers and an environment large enough to avoid trivial cases, which of the following options is advantageous,

(i) Divide the environment $\mathcal{G}$ into $k$ identical parts. Form $k$ groups of $\mathrm{n}$ pursuers each and assign each group to a part of the environment. Run the sweep strategy independently on each group.

(ii) Form a single chain of $k n$ pursuers and search $\mathcal{G}$ using the sweep strategy.

Since the pursuit and capture phases imply capture, the probability of successful evader detection in the sweep phase is equal to the probability of capture of the evader for our sweeping strategy. From the results in Section III, it is evident that larger the total width of the sweep, i.e., $L+l$, the higher is the probability $P$. But, beyond a certain configuration, the convergence time for algorithm TRAP may become arbitrary large. Such a configuration results when $\overline{p_{1} p_{n}}$ is tangent to the sensing discs of all the other pursuers. The separation between the pursuers is $2 r_{\text {step }} \sqrt{\kappa^{2}-4}$.

To obtain finite upper bound on time, we define a limiting formation as under,

Definition V.1 (Limiting trapping chain) Given $a \delta>0$, $n$ pursuers are said to be in the limiting trapping chain if,

(i) for all $k \in\{1, \ldots, n-1\}$,

$$
\left\|p_{k}-p_{k+1}\right\|=2 r_{\text {step }} \sqrt{\kappa^{2}-4} \triangleq d,
$$

(ii) for all $k \in\{2, \ldots, n-1\}$,

$$
\operatorname{dist}\left(p_{k}, \overline{p_{1} p_{n}}\right)=r_{\text {sens }}+\delta \text {. }
$$

Such a limiting configuration is shown in Figure 5. Theorem III.2 leads to the following result for a limiting chain.

Proposition V.2 (Limiting trapping chain property) For a limiting trapping chain,

$$
P(n)=\frac{n d-(3 d-2 c)}{n d-(3 d-l-2 c)} .
$$

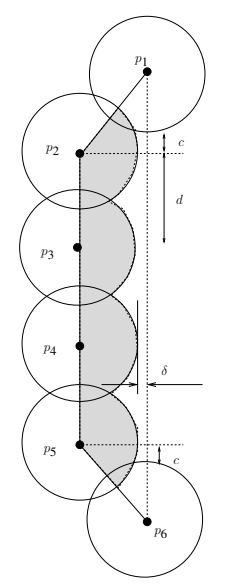

Fig. 5. A limiting trapping chain. The circles around the pursuers represent their sensing discs.

We define a performance metric as the probability of capturing the evader per pursuer, i.e., $\frac{P(N)}{N}$, where $N \triangleq k n$ is the total number of pursuers. Intuitively, this metric refers to the amount of benefit per pursuer. Using option (i) for a limiting trapping chain,

$$
\frac{P(N, k)}{N}=\frac{N d-(3 d-2 c) k}{N(N d-(3 d-l-2 c) k)} .
$$

Equation (3) follows from the fact that when we divide $\mathcal{G}$ into $k$ identical parts, the probability that the evader would be in any one of the parts is $\frac{1}{k}$ and the fact that the capture of the evader by one group implies capture by no other group. The quantity, $\frac{P(N, k)}{N}$, is a maximum when $k=1$. Thus, searching the environment as a conjoined chain is the better option. This fact is supported by a "strong pack adhesive behavior" in wolves, refer Section 14.5 from [19]. 
Let us examine the effect of increasing the size of a single group. Consider a single limiting trapping chain, i.e., $k=1$ and $N=n$. Equation (3) gives us,

$$
\frac{P(n)}{n}=\frac{n d-(3 d-2 c)}{n(n d-(3 d-2 c-l))} \text {. }
$$

The plot of $\frac{P(n)}{n}$ versus $n$ reveals that the measure increases initially, reaches a maximum and then decreases as shown in Figure 6. Thus, there exists an optimal number of pursuers in a single chain. This result is analogous to the results in Model 3 of Packer and Ruttan [16]. This fact is also observed in sizes of wolf-packs which are noted to be ranging from 3 to 15 , refer Section 14.1 from [19].

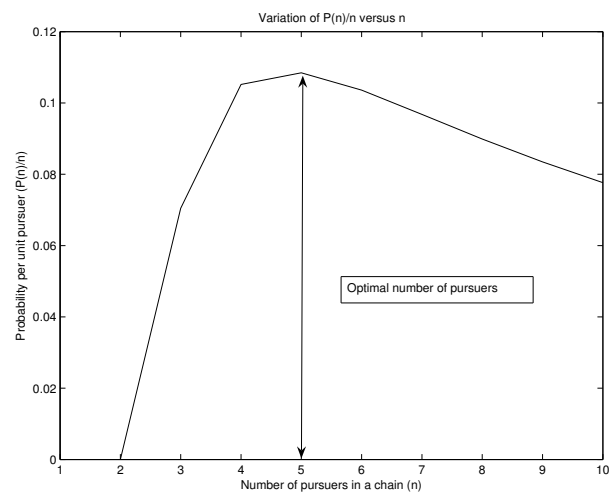

Fig. 6. Plot of $\frac{P(n)}{n}$ versus $n$

\section{CONClusions And Future Directions}

We have investigated the problem of capturing an evader with multiple pursuers in an unbounded environment. We have proposed a sweep-pursuit-capture strategy for a group of pursuers to capture an evader placed randomly inside a bounded region. We gave an expression for the probability of success in the sweep phase which is a function of the pursuer formation and independent of the bounded region and the location of the evader inside it. In the pursuit phase, we proposed a deterministic policy using a novel algorithm TRAP. Finally, we used an existing algorithm SPHERES [5], to complete the capture. Thus, the probability of success in the sweep phase is also the probability of capturing an evader. We then evaluated a decision to be made by a group of pursuers to either conjoin or allocate, during the sweep phase. Using the measure of success probability per pursuer, we concluded that it is advantageous for the pursuers to sweep the region as a conjoined unit. This result has similar analogies in the behavior of wolves during hunting operations.

In this paper, we have considered any arbitrary motion for the evader, once it is detected. In reality, evader motion can be specialized or predictable. We have assumed that the pursuers group together as a chain. Interesting future directions would be to determine and characterize pursuer formations and possibly more efficient strategies for specialized evader behaviors. Additional information on the total time to capture would shed more light on the tendencies to allocate or conjoin.

\section{ACKNOWLEDGMENTS}

This material is based upon work supported in part by ARO MURI Award W911NF-05-1-0219 and NSF SENSORS Award IIS-0330008 and by the Institute for Collaborative Biotechnologies through the grant DAAD19-03-D-0004 from the U.S. Army Research Office. The authors would like to thank Prof. David Skelly for insightful conversations into animal behavior.

\section{REFERENCES}

[1] Y. Ho, A. E. Bryson, and S. Baron, "Differential games and optimal pursuit-evasion strategies," IEEE Transactions on Automatic Control, vol. 10 , no. 4, pp. 385-389, 1965.

[2] S. Lim, T. Furukawa, G. Dissanayake, and H. F. Durrant-Whyte, "A time-optimal control strategy for pursuit-evasion games problems," in IEEE Int. Conf. on Robotics and Automation, New Orleans, LA, Apr. 2004, pp. 3962-3967.

[3] M. Pachter, "Simple motion pursuit-evasion differential games," in Mediterranean Conference on Control and Automation, Lisbon, Portugal, July 2002, Electronic Proceedings.

[4] J. Sgall, "A solution of David Gale's lion and man problem," Theoretical Computational Science, vol. 259, no. (1-2), pp. 663-670, 2001.

[5] S. Kopparty and C. V. Ravishankar, "A framework for pursuit evasion games in $\mathbb{R}^{n}$," Information Processing Letters, vol. 96, no. 3, pp. 114-122, 2005.

[6] L. Alonso, A. S. Goldstein, and E. M. Reingold, "Lion and Man: Upper and lower bounds," ORSA Journal of Computing, vol. 4, no. 4, pp. 447-452, 1992.

[7] S. Alexander, R. Bishop, and R. Ghrist, "Pursuit and evasion in nonconvex domains of arbitrary dimension," in Robotics: Science and Systems, Philadelphia, PA, Aug. 2006, to appear.

[8] S. M. LaValle and J. Hinrichsen, "Visibility-based pursuit-evasion: The case of curved environments," IEEE Transactions on Robotics and Automation, vol. 17, no. 2, pp. 196-201, 2001.

[9] L. J. Guibas, J.-C. Latombe, S. M. LaValle, D. Lin, and R. Motwani, "Visibility-based pursuit-evasion in a polygonal environment," in International Workshop on Algorithms and Data Structures, London, UK, 1997, pp. 17-30.

[10] S. Sachs, S. M. LaValle, and S. Rajko, "Visibility-based pursuitevasion in an unknown planar environment," International Journal of Robotics Research, vol. 23, no. 1, pp. 3-26, 2004.

[11] V. Isler, S. Kannan, and S. Khanna, "Randomized pursuit-evasion in a polygonal environment," IEEE Transactions on Robotics, vol. 5, no. 21 , pp. $864-875,2005$.

[12] B. P. Gerkey, S. Thrun, and G. Gordon, "Visibility-based pursuitevasion with limited field of view," in National Conference on Artificial Intelligence, San Jose, CA, July 2004, pp. 20-27.

[13] V. Isler, S. Kannan, and S. Khanna, "Randomized pursuit-evasion with local visibility," SIAM Journal on Discrete Mathematics, vol. 1, no. 20, pp. 26-41, 2006.

[14] J. P. Hespanha, H. J. Kim, and S. Sastry, "Multiple-agent probabilistic pursuit-evasion games," Electrical Engineering and Computer Science, University of California at Berkeley, Tech. Rep., Mar. 1999.

[15] R. Vidal, O. Shakernia, H. Kim, D. H. Shim, and S. Sastry, "Probabilistic pursuit-evasion games: Theory, implementation and experimental evaluation," IEEE Transactions on Robotics and Automation, vol. 18, no. 2, pp. 662-669, 2002.

[16] C. Packer and L. Ruttan, "The evolution of cooperative hunting," The American Naturalist, vol. 132, no. 2, pp. 159-198, August 1988.

[17] S. K. Gazda, R. C. Connor, R. K. Edgar, and F. Cox, "A division of labour with role specialization in group-hunting bottlenose dolphins (Tursiops truncatus) off Cedar Key, Florida," Proceedings of the Royal Society B: Biological sciences, vol. 272, no. 1559, pp. 135-140, 2005.

[18] B. L. Partridge and T. J. Pitcher, "The sensory basis of fish schools: Relative roles of lateral line and vision," Journal of Comparative Physiology A, vol. 135, no. 4, pp. 315-325, 1980.

[19] J. D. Murray, Mathematical Biology II: Spatial Models and Biomedical Applications, 3rd ed. New York: Springer Verlag, 2004. 